\title{
Radiographic Features of the Maxillofacial Anomalies in Beta-Thalassemia Major: With New View
}

\author{
Soheyla Bayati ${ }^{1}$, Bijan Keikhaei ${ }^{2}$, Mohammad Bahadoram ${ }^{2}$, \\ Mohammad-Reza Mahmoudian-Sani ${ }^{2}$, Mohammad Vaneshani ${ }^{{ }^{*}}$, Fatemeh Behbahani ${ }^{1}$
}

1. Department of Oral \& Maxillofacial Radiology, Dental Faculty, Ahvaz Jundishapur University of Medical Sciences, Ahvaz. Iran

2. Thalassemia \& Hemoglobinopathy research center, Ahvaz Jundishapur University of Medical Science, Ahvaz, Iran

*Corresponding Author:

Mohammad Vaneshani

Department of Oral \& Maxillofacial Radiology, Dental Faculty, Ahvaz Jundishapur University of Medical Sciences, Ahvaz. Iran

Tel.: +98-61-33750410

Email: mohamadvaneshani@gmail.com

Received: 19 May 2021

Revised: 21 Jul 2021

Revised: 25 Aug 2021

\section{ABSTRACT}

\section{BACKGROUND}

Beta- thalassemia major causes the basic skeletal changes due to ineffective erythropoiesis in suffering patients. The aim of the study was to determine the frequency of maxillo-facial anomalies and the hemoglobin and ferritin levels in patients with beta-thalassemia major compared to the healthy control group.

\section{METHODS}

The present study was performed on 72 beta- thalassemia major patients and 70 healthy control group in Ahvaz, Southwest Iran, from Jan 2014 to Mar 2015. Panoramic radiographs were taken using a standard procedure. The frequency of abnormalities including enlargement of bone marrow spaces, small maxillary sinuses, thickness of inferior mandibular cortex, prominent antegonial notch, absence of inferior alveolar canal and thin lamina dura, were determined by two Oral and Maxillofacial Radiologist. We also paid to identification of the relationship between abnormalities frequency and hemoglobin and ferritin levels during previous 6 months in thalassemia patients.

\section{RESULTS}

The mean age of case and control groups was $18.6 \pm 7.25$ and $17 \pm 6.55 \mathrm{yr}$, respectively. The frequency of abnormalities in the case and control groups was as follows, enlargement of bone marrow spaces [69 (95.8\%) vs $3(4.3 \%)$ ], small maxillary sinuses [45 (62.5\%) vs $1(1.4 \%)]$, reduced thickness of inferior mandibular cortex $[21(29.2 \%)$ vs $6(8.6 \%)]$, prominent antegonial notch $[10(13.9 \%)$ vs $2(2.9 \%)]$, absence of inferior alveolar canal [68(94.4\%) vs $41(58.6 \%)$ ] and thin lamina dura [ $40(55.6 \%)$ vs $5(7.1 \%)]$.

\section{CONCLUSION}

The all above mentioned abnormalities in patients with beta-thalassemia major was higher than the control group. Moreover, the frequency of maxillofacial abnormalities decreased by increasing hemoglobin and decreasing ferritin.

\section{KEYWORDS}

Maxillo; Facial anomalies; Beta thalassemia; Blood transfusion

\section{Please cite this paper as:}

Bayati S, Keikhaei B, Bahadoram M, Mahmoudian-Sani MR, Vaneshani M, Behbahani F. Radiographic Features of the Maxillofacial Anomalies in BetaThalassemia Major: With New View. World J Plast Surg. 2021;10(2):78-83. doi: $10.29252 /$ wjps. 10.3 .78 


\section{INTRODUCTION}

Thalassemia is the most common known single gene disorder in the wide world, which has a variety of types ${ }^{1}$. Beta-thalassemia major is the most severe form of beta thalassemia which can be lifethreatening, if remains untreated. Iran and other Middle East countries are the endemic areas for this disease. In Iran there are 25,000 patients with betathalassemia major ${ }^{1,2}$.

The etiology of this disease is a defect in hemoglobin beta protein synthesis that leads to reduced hemoglobin, increased ferritin level, extensive hemolysis, and iron overload ${ }^{3}$. 4. Therefore, hyperplasia and maxillofacial expansion develops that can lead to maxillofacial deformities ${ }^{5,}{ }^{6}$. The impact of thalassemia on the jaw and skull radiograph include decreased of general bone density due to bone marrow overgrowth, thin cortical bone, absence of inferior alveolar canal, and small maxillary sinuses ${ }^{7,8}$.

Blood transfusion as a key treatment is applied to prevent of the skeletal changes in patients. Hemoglobin concentration must be higher than 10 for arrived to this aim ${ }^{9}$. Unfortunately, despite numerous clinical studies on patients with changes in the maxillofacial area, a few investigations had studied maxillo-facial radiological abnormalities. On the other hand, to the best of our knowledge, our study had the largest sample size comparison with other studies. It also appears to be no study has been paid directly to the relationship between hemoglobin and ferritin concentrations with the maxillo-facial abnormalities.

We aimed to compare the frequency of maxillofacial abnormalities and serum levels of hemoglobin and ferritin in patients with beta-thalassemia major with the control group.

\section{MATERIAL AND METHODS}

\section{Ethics Approval}

This trial was approved by the Ahvaz Jundishapur University of Medical Sciences Ethics Committee (approval no: IR.ajums.REC.1393.276). Informed consent was taken from all participants in accordance with the provisions of the Declaration of Helsinki, after approval of the Ethics Committee of Ahvaz Jundishapur University of Medical Sciences.

This case-control study was performed in Ahvaz,
Southwest Iran, from Jan 2014 to Mar 2015. The case group included 72 patients of definitive diagnosis of beta-thalassemia major under transfusion therapy on a monthly basis (according to the treatment protocol). The control group also was selected from individuals referred to the Oral Medicine and Radiology Department of Ahvaz Jundishapur University of Medical Sciences. Inclusion criteria were no history of blood diseases or syndromes. Demographic data and the mean serum hemoglobin and ferritin concentrations of patients during past 6 months were collected from the medical records. The panoramic radiographs were taken in both case and control group. They were prepared with cranex D (PM 2010, Finland, Sordex). The evaluation of radiographic images was performed on the monitor (SONY VAIO D13213CX) by two oral radiologists, separately. The radiographs were examined for any radiological abnormalities include enlargement of bone marrow spaces, small maxillary sinuses, thickness of inferior mandibular cortex, prominent antegonial notch, and absence of inferior alveolar canal and thin lamina dura.

Types of abnormalities were classified by the definitions of Hazza et al.s study10 except for thin lamina dura as White and Pharoah textbook definition11. In addition, we indicated the effects of gender, hemoglobin and ferritin concentrations on maxillo-facial radiologic abnormalities in the form of a probabilistic model proposed by regression logistic model, for the first time. Investigators (oral radiologists) reviewed together again radiographs in cases where there was disagreement among them. Finally, observers agreed on all graphics interpretations and reported the same interpretations. The significant level was considered as $P<0.05$. The Chi-Square test was used to compare the relative frequency of each parameter in the control and case groups. Data were analyzed using SPSS 18 (Chicago, IL, USA).

\section{RESULTS}

Among 72 patients, $29(40.3 \%)$ were female and 43 (59.7\%) were male. Similarly, 70 patients were entered to the control group. There was a similar distribution of age and gender in both groups (Table $1)$.

The patients were divided into 3 subgroups ages, including less 10, 10-20, and higher 20 
Table 1: Age and sex distribution of the case and control group

\begin{tabular}{ccccc}
\hline \multicolumn{2}{c}{ Variable } & Beta thalassemia major & Control & P-value \\
\hline \multirow{2}{*}{ Gender } & Female (\%) & $29(40.3)$ & $34(48.6)$ & 0.32 \\
& Male (\%) & $43(59.7)$ & $36(51.4)$ & \\
& Age & $18.57 \pm 7.25$ & $16.97 \pm 6.55$ & 0.17 \\
\hline
\end{tabular}

Table 2: The frequency and percent of radiographic deformities in patients with major beta thalassemia compared with the control group

\begin{tabular}{lccc}
\hline Radiographic deformities & Beta- thalassemia major N(\%) & Control N(\%) & $P$-value \\
\hline Enlargement of bone marrow spaces & $69(95.8)$ & $3(4.3)$ & $<0.001$ \\
Absence of inferior alveolar canal & $68(94.4)$ & $41(58.6)$ & $<0.001$ \\
Small maxillary sinuses & $45(62.5)$ & $1(1.4)$ & $<0.001$ \\
Thin lamina dura & $40(55.6)$ & $5(7.1)$ & $<0.001$ \\
Reduced thickness of inferior mandibular cortex & $21(29.2)$ & $6(8.6)$ & 0.002 \\
Prominent antegonial notch & $10(13.9)$ & $2(2.9)$ & 0.03 \\
\hline
\end{tabular}

Table 3: Relationship between the median of hemoglobin and ferritin serum and maxillofacial abnormalities in patients with major

\begin{tabular}{lcccc}
\multicolumn{5}{c}{ beta thalassemia } \\
\hline Type of Anomaly & $\mathrm{Hb}$ & P-Value & Ferritin & $P$-value \\
\hline Enlargement of bone marrow spaces & $7.85(6.2-11.6)$ & 0.22 & $3100(149-5950)$ & 0.46 \\
Thin lamina dura & $7.85(6.2-11.6)$ & 0.42 & $3100(200-5950)$ & 0.003 \\
Small maxillary sinuses & $7.85(6.20-11.30)$ & 0.95 & $3110(375-5147)$ & 0.96 \\
Reduced thickness of inferior & $7.70(6.20-9.60)$ & 0.31 & $3247(1000-5147)$ & 0.48 \\
mandibular cortex & $7.65(6.80-8.70)$ & 0.20 & $3041(1000-4900)$ & 0.77 \\
Prominent antegonial notch & $7.78(6.20-11.60)$ & 0.30 & $3100(149-5950)$ & 0.47 \\
Absence of inferior alveolar canal & & & & \\
\hline
\end{tabular}

Table 4: The logistic regression model for Enlargement of bone marrow spaces based on age, gender, $\mathrm{Hb}$ and ferritin

\begin{tabular}{lccc}
\hline Enlargement of bone marrow spaces & $\beta$ & Sig & Odds Ratio \\
\hline Age & 0.32 & 0.048 & 1.38 \\
$\mathrm{Hb}$ & 0.57 & 0.53 & 1.76 \\
Ferritin & -0.001 & 0.18 & 0.99 \\
gender & -0.89 & 0.55 & 0.41 \\
\hline
\end{tabular}

yr of age. There was no statistically significant relationship between the age and frequency of maxillo-facial abnormalities in three age groups. Moreover, there was no significant difference between the maxillo-facial abnormalities and gender of patients. The most frequency of maxillafacial deformities, in patients with beta- thalassemia major vs. control group are shown in Table 2. There was no significant correlation between the median hemoglobin and maxilla-facial abnormalities (Table 3). Similarly, there was no significant relationship between the mean serum ferritin and maxillofacial deformities, except thin lamina dura anomaly. We also used logistic regression model for prediction the occurrence of types of anomalies based on different factors. These factors included age, gender, hemoglobin, and ferritin concentrations (Table 4-9). The probability risk for the enlargement of bone marrow spaces in patients increased by $38 \%(\mathrm{OR}=1.38)$ per each year increasing the age. The occurrence of reduced thickness of inferior mandibular cortex abnormality was 2.2 more frequent in males than females $(\mathrm{OR}=0.44)$. In addition to, thin lamina dura was 2.3 more frequent in females $(\mathrm{OR}=2.34)$. Moreover, for each 1-unit decrease in hemoglobin level, the chance of reduced thickness of inferior mandibular cortex, absence of inferior alveolar canal, and prominent antegonial 
Table 5: The logistic regression model for thin lamina dura based on age, gender, $\mathrm{Hb}$ and ferritin

\begin{tabular}{lccc}
\hline Thin lamina dura & $\beta$ & $\mathrm{Si}$ & OR \\
\hline Age & & $\mathrm{g}$ & 1.02 \\
$\mathrm{Hb}$ & 0.02 & 0.58 & 1.38 \\
Ferritin & 0.32 & 0.23 & 1 \\
gender & 0.0 & 0.47 & 2.34 \\
\hline
\end{tabular}

Table 6: The logistic regression model for Small maxillary sinuses based on age, gender, $\mathrm{Hb}$ and ferritin

\begin{tabular}{lccc}
\hline Small maxillary sinuses & $\beta$ & sig & OR \\
\hline Age & -0.042 & 0.26 & 0.959 \\
$\mathrm{Hb}$ & 0.005 & 0.98 & 1.005 \\
Ferritin & 0.0 & 0.83 & 1.00 \\
gender & -0.38 & 0.46 & 0.685 \\
\hline
\end{tabular}

Table 7: The logistic regression model for Reduced thickness of inferior mandibular cortex based on age, gender, Hb and ferritin.

\begin{tabular}{lccc}
\hline Reduced thickness of inferior mandibular cortex & $\beta$ & sig & OR \\
\hline Age & -0.027 & 0.49 & 0.973 \\
$\mathrm{Hb}$ & -0.3 & 0.29 & 0.74 \\
Ferritin & 0.0 & 0.42 & 1 \\
gender & -0.806 & 0.16 & 0.447 \\
\hline
\end{tabular}

Table 8: The logistic regression model for Prominent antegonial notch based on age, gender, $\mathrm{Hb}$ and ferritin

\begin{tabular}{lccc}
\hline Prominent antegonial notch & $\beta$ & Sig & OR \\
\hline Age & 0.035 & 0.51 & 1.036 \\
$\mathrm{Hb}$ & -0.62 & 0.16 & 0.54 \\
Ferritin & 0.0 & 0.97 & 1 \\
gender & -0.546 & 0.44 & 0.58 \\
\hline
\end{tabular}

Table 9: The logistic regression model for Absence of inferior alveolar canal based on age, gender, Hb and ferritin

\begin{tabular}{lccc}
\hline Absence of inferior alveolar canal & $\beta$ & sig & OR \\
\hline Age & -0.074 & 0.38 & 0.93 \\
$\mathrm{Hb}$ & -0.394 & 0.37 & 0.67 \\
Ferritin & 0.0 & 0.59 & 1 \\
gender & -1.002 & 0.41 & 0.367 \\
\hline
\end{tabular}

notch increased 35\% (OR=1.35), 49\% (OR=1.49), and $85 \%(\mathrm{OR}=1.85)$, respectively.

\section{DISCUSSION}

The nature of beta-thalassemia major is in a manner that may cause major changes in morphology especiallyin maxillofacialorgans as part of the skeletal system. Although, maxillofacial malformations are well known in patients with beta-thalassemia major, a few studies have been performed on the frequency of radiological abnormalities of patients ${ }^{10,12}$. Unlike some studies on radiographic images of thalassemia, examiners decided after were agreed together in all the details of the results give the unit results of their observation.

We decided to use this method since the investigated abnormalities in our study are more visible than other abnormalities and the reading of the investigated abnormalities is relatively easy. 
This method may be more efficient compared to existing and conventional methods that examiners have nothing relationship together. This is because after the observer's arguments against the agreement after they have seen what will be done. In this study, the observers on all the details of the abnormalities are found to agree $100 \%$, which in itself is new. Furthermore, few studies had examined the maxillofacial deformities in individuals older than 18 yr in patients with thalassemia major; and our study had the largest sample size comparison with other studies $^{12}$. In addition, to the best of our knowledge, we studied the relation between frequency of these abnormalities with the median ferritin and hemoglobin level during the last 6 months ago, in the patients affected, for the first time. According to the present study, maxilla-facial malformations had the highest frequency, in the enlargement of bone marrow spaces, absence of inferior alveolar canal, small maxillary sinuses, thin lamina dura, reduced thickness of inferior mandibular cortex, prominent antegonial notch, respectively. With little difference, Hazza and Jamal introduced reduced thickness of inferior mandibular cortex, absence of inferior alveolar canal, small maxillary sinuses, thin lamina dura, enlargement of bone marrow spaces and prominent antegonial notch as the highest frequency, respectively ${ }^{10}$.

In our study, thin lamina dura was seen in 40 (55.6\%) patients with thalassemia and in 5(7.1\%) patients of the control group. This result was concordance with other studies that reported deformity in $46 \%$ and $40 \%$ of patients with thalassemia, respectively ${ }^{10}$, 12. Small maxillary sinuses were reported in 45 thalassemic patients (62.5\%) and in $1(1.4 \%)$ individual in the control group in our study. Similarly, in some studies were seen in 50\% and 56\% of patients with thalassemia, respectively ${ }^{10,12}$. The prominent antegonial notch in our study was in 10 (13.9\%) patients with thalassemia and in 2 (2.9\%) samples of the control group. That in accordance with our results, in another study 9 (18\%) of patients with thalassemia and 7 (14\%) of the control group had abnormalities, respectively ${ }^{10}$. Although Hazza and Jamal introduced the reduced thickness of inferior mandibular cortex in all patients with thalassemia major, we had a completely contradict their findings. In our study, it was observed only in $21(29.2 \%)$ samples $^{10}$. Probably part of the difference was for this reason that we considered the values less than $3 \mathrm{~mm}$ as the reduced thickness of inferior mandibular cortex in adults (9) in contrast to other studies $^{10,12}$.

Complete absence of inferior alveolar canal was seen in $33(66 \%)$ patients with thalassemia and in $23(46 \%)$ cases in other studies. Contrast to this finding, we demonstrated $68(94.4 \%)$ of cases had this abnormality. The difference in this study may be related to this fact that the higher incidence of this disease in our studied population. Because in healthy subjects of our study, 41 (58.6\%) samples had these disorders. However, this deformity was seen another study only in $1(2 \%)$ of the control group ${ }^{10,12}$. The enlargement of bone marrow spaces was introduced in $21(42 \%)$ patients with thalassemia and others reported this number, 27 (54\%). However, in our study, 69 (95.8\%) patients, compared with 3 (4.3\%) of the control group showed this abnormality ${ }^{10}$, ${ }^{12}$. This can be contributed to higher age mean of individuals. Because the mean age of patients in our study was $18.57 \pm 7.25 \mathrm{yr}$ and significantly higher than in other studies $\left(7.15 \pm 0.9^{10}, 14.34 \pm 5.83^{12}\right)^{\prime}$ this may explain the results ${ }^{11}$. However, in our study, there was not a significant correlation between age and any maxillo-facial abnormalities. This can be related to higher frequency of individuals in the age group 10-20 yr in subjects.

The thalassemia causes wide systemic changes on skeletal system and the various body organs ${ }^{13,14}$. However, it does not seem like a study to examine the relationship between hemoglobin and ferritin and maxillofacial anomalies. The effect of blood transfusion regimen (based on amount of blood transfused) was evaluated on the head and teeth in children with severe thalassemia. According to this study, most patients with beta-thalassemia major who received a high blood transfusion regimen had lower anomalies rate than low blood transfusion regimen ${ }^{15}$.

\section{CONCLUSION}

In our study, there was no significant relationship among the frequency of maxillofacial anomalies and mean hemoglobin and ferritin. This may be related to lower sample size of individuals with low hemoglobin. There was a significant relationship between blood transfusion regimen and reduction of maxillofacial anomalies. Moreover, the frequency of maxillo-facial abnormalities decreased by 
increasing hemoglobin and decreasing ferritin.

\section{ACKNOWLEDGEMNTS}

We gratefully thank Research and Technology Deputy of Ahvaz Jundishapur University of Medical Sciences for funding the project (grant no. th93/16).

\section{CONFLICT OF INTEREST}

The authors declare that there is no conflict of interest.

\section{REFERENCES}

1 De Sanctis V, Kattamis C, Canatan D, et al. $\beta$-Thalassemia Distribution in the Old World: an Ancient Disease Seen from a Historical Standpoint. Mediterr J Hematol Infect Dis 2017;9(1):e2017018-e. https://pubmed.ncbi.nlm.nih.gov/28293406/

2 Khodaei GH, Farbod N, Zarif B, Nateghi S, Saeidi M. Frequency of thalassemia in Iran and Khorasan Razavi. Int J Pediatr 2013;1(1):45-50. https://ijp. mums.ac.ir/article_2044.html

3 Rechavi G, Rivella S. Regulation of iron absorption in hemoglobinopathies. Curr Mol Med 2008;8(7):64662. https://pubmed.ncbi.nlm.nih.gov/18991651/

4 Nienhuis AW, Nathan DG. Pathophysiology and Clinical Manifestations of the $\beta$-Thalassemias. Cold Spring Harb Perspect Med 2012;2(12):a011726-a. https://pubmed.ncbi.nlm.nih.gov/23209183/

5 Helmi N, Bashir M, Shireen A, Ahmed IM. Thalassemia review: features, dental considerations and management. Electron Physician 2017;9(3):40038. https://pubmed.ncbi.nlm.nih.gov/28461877/

6 Amirabadi F, Saravani S, Miri-Aliabad G, KhorashadiZadeh M. The Association between Dental Health Status and Oral Health-Related Quality of Life of Children Diagnosed with beta-Thalassemia Major in
Zahedan City, Iran. Int J Pediatr 2019;7(2):8985-91. https://ijp.mums.ac.ir/article_11475.html

7 Hazza'a A, Al-Jamal G. Radiographic features of the jaws and teeth in thalassaemia major. Dentomaxillofac Radiol 2006;35(4):283-8. https://pubmed.ncbi.nlm. nih.gov/16798927/

8 Valizadeh N, Farrokhi F, Alinejad V, et al. Bone density in transfusion dependent thalassemia patients in Urmia, Iran. Iran J Ped Hematol Oncol 2014;4(2):6871. https://pubmed.ncbi.nlm.nih.gov/25002928/

9 Liumbruno G, Bennardello F, Lattanzio A, Piccoli P, Rossetti G. Recommendations for the transfusion of red blood cells. Blood Transfus 2009;7(1):49-64. https://pubmed.ncbi.nlm.nih.gov/19290081/

10 Hazza’a AM, Al-Jamal G. Dental development in subjects with thalassemia major. J Contemp Dent Pract 2006;7(4):63. https://pubmed.ncbi.nlm.nih. gov/16957792/

11 White SC, Pharoah MJ. White and Pharoah's Oral Radiology E-Book: Principles and Interpretation: Elsevier Health Sciences; 2018.

12 Kashid AL, Kumbhare S, Sathawane R, Mody R. Comparative evaluation of radiographic features of jaws and teeth on opg (orthopentamogram) in thalassemia major patients and normal individuals. Int J Curr Res 2013;5(15):12.

13 Cutando SA, Gil MJ, López-González GJD. Thalassemias and their dental implications. Med Oral 2002;7(1):36. https://pubmed.ncbi.nlm.nih. gov/11788807/

14 Amini F, Jafari A, Eslamian L, Sharifzadeh S. A cephalometric study on craniofacial morphology of Iranian children with beta-thalassemia major. Orthod Craniofac Res 2007;10(1):36-44. https://pubmed.ncbi. nlm.nih.gov/17284245/

15 Jirarattanasopa V, Hooncharoen P, Mekaewkunchorn A, Torcharus K. Effect of different transfusion regimens on craniofacial appearance and dentition in severe thalassemic children. Southeast Asian J Trop Med Public Health. 2009;40(6):1371-6. 